\title{
The Development of Model-Based Learning in Introductory Physics: The effectiveness of improving Students Understanding in Heat and Heat Transfer
}

\author{
Ika Mustika Sari ${ }^{1}$, Saeful Karim² ${ }^{2}$, Duden Saepuzaman ${ }^{3}$, Taufik R. Ramalis ${ }^{4}$, Dadi \\ Rusdiana $^{5}$ \\ \{ikams@upi.edu $\left.{ }^{1}\right\}$ \\ Department of Physics Education, Science and Mathematics Education Faculty, Universitas \\ Pendidikan Indonesia, Jln. Dr. Setiabudhi No. 229 Bandung, West Java, Indonesia, 40154 1,2,3,4,5
}

\begin{abstract}
Heat and heat transfer is still becoming a difficult concept for students due to its characteristic as an abstract concept. This fact leads students to have a misconception about the topic. Introductory Physics as a mandatory subject to Pre-service Physics Teacher has an important role in constructing and developing students' scientific concept. Model-based learning (MBL) was developed and has been implementing in this course. Design-based research as a research method was used in examining the effectiveness of MBL. Results show that MBL has an impact on improving students' understanding of heat and heat transfer. Both the effect size and normalized gain showed a positive increase in the medium category.
\end{abstract}

Keywords: Model-based Learning, Heat, Heat Transfer, Introductory Physics

\section{Introduction}

Physics is a fundamental course and is one of the branches of science that learns the broad of objects both observable directly without aided by the tools and unobservable ones. The unobservable objects in physics can be called as abstract objects. To comprehend the behavior and/or the properties of abstract objects, imagining and providing the external representation are necessary to be conducted. The examples of the abstract object are atoms, molecules, energy, solar system, galaxy, etc. Due to it contains abstract objects, Physics is often considered as a difficult subject both at school level and at college-level [1], [2], [3].

One of the physics concepts which is hard to be understood and abstract is a concept that relates to thermodynamics: heat and heat transfer. It is also found as a concept that has many misconceptions [4], [5], [6], [7], [8]. On the other hand, heat and heat transfer are important concepts that should be mastered by students [9], [10]. Heat and heat transfer as a mandatory topic is learned in school start from junior high school to college. Physics teachers must have a scientific comprehension of this topic. Therefore, Education universities that produce physics teacher candidates faces a challenge to train their physics teachers candidate so that the students master the topic and have a good and scientific understanding of heat and heat transfer concepts.

In order to improve teacher students ' comprehension in heat and thermodynamic, it needs refinement and continual improvement in the learning process both at school level and university level especially in institutions that graduate the physics teachers' candidate. Teachers 
in these institutions (we can call Lecturer) play an important role in improving the quality of physics learning. The lecturer must conduct the research and generates recommendations in refining methods, learning models, learning materials, etc.

When a lecturer is conducting the improvement of the quality of learning through learning models innovation and research, they actually are improving the quality of physics learning not only in the university-level but also at all educational levels. We can understand it as the research subject is teachers candidates, and immediately they will be physics teachers who have skills and scientific understanding. Furthermore, we can conclude that research that relates to the developing of learning models in the Introductory Physics course to improve physics teachers“ candidates' understanding of heat and heat transfer, is necessary to be conducted.

Based on the previous researches, there are many misconceptions found in heat and thermodynamics concepts. The misconception is a students' conception that unscientific and is believed as correct conception. The misconception is often referred to as alternative conception because misconception points to the existence of students owned models or frameworks of thinking that differ from (alternate form) the scientific theory [9]. If misconception does not to be well overcome, it can be a belief or concept that they correctly think. One of the causes that there is a misconception in the heat concept is students think that heat is not a form of energy. They failed to comprehend that heat is like a concept of work in mechanics. Heat is transfer energy due to the difference in temperature between two or more systems that are thermally contacted. Many students think that heat is materials/molecules that spread or flow from higher temperature objects to the lower one while they are thermally contacted, as a consequence, higher temperature object contains more heat rather than the lower temperature object [11]. Other misconceptions in heat concept: heat is viewed as an object property, like wood is hot, while ice is cold[11]. Another misconception of heat is that students are failed to distinguish between heat and internal energy, heat, and temperature, therefore students have a difficulty in identifying the interaction of heat and work of system and environments [7].

One of the alternative solutions to overcome the problems stated above is implementing the learning models that proved to improve students' understanding and minimize students' misconceptions. MBL has a huge potential in supporting students to construct scientific comprehension [12], [13]. Model-based learning (MBL) is proposed as one learning model that provides scientific models and facilitates students' construction of scientific understanding through the modeling process. Scientific models presented in MBL help students to imagine an abstract concept such as heat, temperature, and energy. Another characteristic of MBL is the modeling process in which students are given the opportunity to construct scientific models through model construction [13]. Students get used to actively think to develop their own models to answer the problems addressed by the teacher.

The process of developing MBL begins with identifying students' understanding through tests and semistructured interviews to elicit students' mental models of phenomenon relates to heat conduction and heat convection [14], [15], [16], [17]. Based on the results of that research, we can conclude that pre-service physics teachers have a lack of knowledge, many misconceptions and un-scientific mental models of heat and heat transfer concepts. Therefore, research, in order to improve students' comprehension of heat and heat transfer concepts, is a must. Research questions are as follows:

1. What the impact of MBL implementation on students ' understanding of heat and heat transfer concepts?

2. How the improvement of students ' understanding of heat and heat transfer after they are intervented by $M B L$ ? 


\section{Model-based Learning}

Model-based learning can be called as a strategy to improve students " understanding by providing students with scientific models and facilitates students to construct scientific models.

\subsection{Models in Physics Education}

Models in Physics (Science) Education. Models in Physics is described as a representation of structure in a physical system and/or its properties [2]. Models are used in Physics education for a specific purpose e.g to bring the abstract concepts into observable concepts. Models provide an application of the knowledge to real-world situations-made to see how things apply in the real world instead of just looking at equations [2]. There are many kinds of models in Physics Education such as mental models; conceptual models which are mathematical models, computer models, and physical models; and physics models [2].

Mental Models. Mental models are internal representations, which are located in mind and are generated from a person perceives and conceptualize the situations happening in the world [2].

Conceptual Models. Conceptual models are an external representation created by teachers or scientists that facilitates the comprehension or the teaching of systems or states of affairs in the world [24]. Conceptual models are simplified and idealized representations of real objects, phenomena, or situations [2]. Examples of conceptual models are the concept of water pump as a model for the battery in an electrical circuit; Solar system model as a conceptual model of an atom. Since mathematical models, computer models and physical models are an external representation and are made by teachers and experts, therefore, mathematical models, computer models, and physical models are categorized as conceptual models.

Physics Model. A physics model in the physics-education community is considered as a simplified and idealized physical system, phenomenon, or idealization. For instance, a particle is a physics model for the object in motion learning in which the shape of it is far less rather than the length of path along with its motion. Another example of a physics model is A simple pendulum because it is idealized and consists of a mass particle on a massless string on invariant length moving in the homogenous gravitational field of the Earth in the absence of drag due to air [2].

\subsection{Model-based Learning}

Models based learning (MBL) is an approach in the science learning process in which learning is conducted by construction models as a representation of physics phenomena, includes representation of physics objects and its characteristics, physics entities and physics process that involved physics phenomena [13].

\subsection{Misconception in Heat and Heat Transfer}

Many types of research revealed and have explored the existence and resilience of students various unscientific understandings about physical phenomena especially phenomena relates to heat and thermodynamics. Yeo and Zadnik documented some general findings of students ${ }^{6}$ thermal understandings learned from the research are as follows [5]: (1). Many conceptions are context-dependent and explanations are related to single or isolated situations; (2). Students are 
inconsistent in their explanations; (3). Students are more likely to express alternative conceptions when explaining real-life situations; (4). Students' knowledge frameworks often allow them to accept a statement of what is as a sufficient explanation of why; (5). Students often admit to being unclear about their ideas even they make correct answers. The main misconception of Heat is often referred to as materials that has hot property, therefore, some students think that heat is contained by objects [11], [14], [15], [16], [17]. Students are failed to explain that heat is a form of energy and has an analogy to work in mechanics. Whereas, heat is energy transfer that exists when two objects have different temperature and they are thermally contacted. Another finding of misconception in heat and the thermodynamic concept is temperature measures the heat content of the body [4].

When students try to answer and explain the phenomena relates to heat and thermodynamic, they are often influenced by intuitive thinking or common sense without concerning the scientific concept. This is one of the spirit in conducting research in improving students understanding and minimizing students ' misconceptions in heat and thermodynamic concepts.

\section{Research Method}

In this research, we developed a Models that facilitates students to construct their scientific models and providing scientific models through Design Based Research (DBR). DBR is a quite new and promising methodology that focused on research of developing learning models, include developing syntax of learning [18]. DBR as a methodology is developed and designed in early 21 st Century by and for educators. DBR is practical methodology that can be effectively bridged the discrepancy between research and practice in formal education [19]. Even DBR is often called as a new methodology, DBR is convinced has a great potential in making significant difference from the aspect of quality and utilization of educational research results [20]. The main goal of DBR is to seek the impact of improvement, to transfer and to translate educational research into the educational practice .

1. The quality of DBR is determined by [19]:

2. Is situated in real-context education.

3. Focused on the design and examining the significance of intervention.

4. Using mixed methods in assessing the intervention of DBR.

5. Involving repetition literacy.

6. Involving the partnership of collaboration between researcher and practitioner.

7. Developing the principles of design.

Research in developing MBL in Introductory of Physics Course utilize DBR methodology was conducted in several steps as follows: (1) Identification of misconception and mental models in heat and heat transfer; (2) Designing of learning model; (3). Implementation of developed learning model; (4) Examining the effectiveness of the developed learning model; (5). Analysis and Revise of developed learning model; (6). Re-implementation of developed learning; (7). Re-examining the effectiveness of the developed learning model; (8). Final design of the developed learning model: MBL that enable to improve students' understanding of heat and heat transfer. In this article, researchers will report the effectiveness of MBL in improving students' understanding of heat and heat transfer concepts.

Mixed methods used to assess the effectiveness of MBL: Effect size and normalized gain from the quantitative aspect; while the process of drawing and construction students ' models in heat and heat transfer from the qualitative aspect. In this article, an analysis of the effectiveness 
of the Model-based learning developed by using DBR is more focused on the results of processing the effect size and normalized gain data.

\section{Results and Discussion}

The first phase of this research after analyzing the students' mental model through semistructure interview was designing the learning model. The learning model that we used in this phase is Model based learning that consist of two characteristics: (1) presented scientific models such as animations of particles that have higher temperature from Phet Simulations, picture of Molecules models in solids substance, demonstration of expansion in solids and convection in boiling water; (2). Facilitated students' construction of scientific models (modelling process) through drawing of moleculles composition/ structure when they are heated. The modelling process was guided by students worksheet. Students worked together in groups that consist of 5-7 persons. To test the effectiveness of this treatment, we calculate the normalized gain, statistical test and effect size as it follows.

\subsection{Normalized Gain}

Normalized Gain. Normalized gain is used in calculating the increase in understanding of the concept of heat and its propagation after the implementation of MBL. The calculation of normalized gain is based on the equation (1) that is developed by Meltzer [22]:

$$
<g>=\frac{\text { postest score-pretest score }}{\text { ideal score-pretest score }}
$$

Normalized gain calculation results are then interpreted using the classification categories made by Hake [23]. Based on the results of data analysis, it was found that the normalized gain of 0.41 included in the medium category.

\subsection{Statistical testing on improving understanding of concepts}

Test of Normality. Test of Normality of pretest and posttest data was conducted by using Kolmogorov-Smirnov test statistic with the hypothesis: If the value of significance (p-value) < 0,05 , then data is not normally distributed. Inversely, If the value of significance (p-value) $>=$ 0,05 , then data is normally distributed. The test of normality is shown in Table 1.

Table 1. Test of Normality.

\begin{tabular}{lcccccc}
\hline & \multicolumn{3}{c}{ Kolmogorov-Smirnov } & \multicolumn{3}{c}{ Shapiro-Wilk } \\
& Statistic & df & Sig. & Statistic & df & Sig. \\
\hline PRE & .189 & 36 & .002 & .888 & 36 & .002 \\
POS & .305 & 36 & .000 & .667 & 36 & .000 \\
T & & & & & & \\
\hline
\end{tabular}

a. Lilliefors Significance Correction

Based on Table 1, we can see that the significance of pretest and posttest data Sig. $<0.05$, we can conclude that the data is not normally distributed. 
In the next step, we will check the homogeneity of variance, whether or not the variance was homogen. The result of test homogeneity was shown in Table 2.

Table 2. Test of Homogeneity of Variance.

\begin{tabular}{llcccc}
\hline & & $\begin{array}{c}\text { Levene } \\
\text { Statistic }\end{array}$ & df 1 & df 2 & Sig. \\
\hline Result & Based on Mean & .0007 & 1 & 70 & .936 \\
& Based on Median & 0.39 & 1 & 70 & .844 \\
& Based on Median and with & .039 & 1 & 63.752 & .844 \\
& adjusted df & 0.16 & 1 & 70 & .898 \\
\hline
\end{tabular}

Based on Table 2, we can see that the significance of homogeneity variance Sig. > 0.05, therefore, we can conclude that the data is homogenous.

The next step is the hypothesis test. Based on the result of previous analysis, the testing of hypothesis uses the Wilcoxon Signed Ranks Test. The result of the test was shown in Table 3.

Table 3. Test Statistic ${ }^{a}$

\begin{tabular}{ll}
\hline & POST-PRE \\
\hline $\mathrm{Z}$ & $-3.010^{\mathrm{b}}$ \\
Asymp. Sig. (2-tailed) & .003 \\
\hline \multicolumn{2}{c}{ a. Wilcoxon Signed Ranks Test } \\
b. Based on Negative Ranks
\end{tabular}

Based on the result shown on Table 3, we can conclude that there is a significant difference between post treatment and pre-treatment. Therefore, Model Based Learning has an impact to improve students' understanding on heat and heat transfer concept.

\subsection{Effect Size}

Effect size. Effect size is a measure of the practical significance of research results in the form of a measure of correlation or difference, or the effect of a variable on other variables show on equation (2). Effect size is calculated by Cohen's formula d, as follows:

$$
d=\frac{x_{1}-\underline{x_{2}}}{\underline{S_{d e v}}}
$$

in which:

$\underline{x_{1}}:$ the average of posttest score

$x_{2}:$ the average of pretest score

Therefore, based on the results of the calculation of the posttest and pretest values, an effect size value of 0.69 is obtained and this is included in the medium category.

Based on the results obtained in 4.1, 4.2, and 4.3 we can see that Model-Based Learning, that designed through DBR can effectively improve students' understanding of the concepts of heat and heat transfer. However, although it increased significantly based on the statistical 
calculations, the increase was still in the moderate category. In addition, the effect size obtained from the effect size calculation also has a moderate effect size. This can be used as a baseline in the second phase of developing MBL based on the findings in Phase 1.

\subsection{Students ${ }^{6}$ mental models and responses}

Students' activity in constructing models utilized the function of whiteboard through the modelling process that is called the white-boarding process. Students was divided into six groups and each of group was equipped with whiteboard and markers. Students was guided by worksheet that encouraged students to elicit their own ideas about the structure of matters before and after heating process. In the phase of reinforcement, each of groups presented what they found enriched with scientific arguments. In the last phase, teacher opened the discussion session and confirmed the findings with the scientific views and theory.

Students' responses to the implementation of MBL were welcomed and documented by the teacher. Most of the students expressed their excitement involving in learning through Model based learning.

\section{Conclusion}

This research revealed that the implementation of developed MBL through DBR has a positive impact and significantly improve the students' understanding of heat and heat transfer concept. The improvement is still in the medium category and it necessary to be continued to phase 2 .

\section{References}

[1] Halloun, I.: Schematic Modeling for Meaningful Learning of Physics. Vol. 33 (9), pp. 1019-1041. Journal of Research in Science Teaching (1996)

[2] Ornek, F., Robinson, W.R. and Haugan, M.P.: What makes physics difficult? Vol. 3 (1), pp. 30-34 International Journal of Environmental \& Science Education. (2008).

[3] Ornek, F., Robinson, W.R. and Haugan, M.P.: What makes physics difficult?. Vol. 18 (3) pp. 165-

172. Science Education International (2007)

[4] Pathare, S. and Pradhan, H.C.: Students' Alternative Conceptions in Pressure, Heat and Temperature. Pp. 38-41. Trends in Science Education Research. (2005)

[5] Yea, S. And Zadnik, M.: Introductory thermal concept evaluation. Vol. 39 pp. 496-501. The Physics Teacher (2001)

[6] Pathare, S. and Pradhan, H. C.: Students' Alternative Conceptions in Pressure, Heat and Temperature. Pp. 38-41. Trends in Science Education Research (2005)

[7] Miller, R. L., Streveler, R. A., Nelson, M. A., Geist, M. R., Olds, B. M. .: Developing an instrument to measure engineering student misconceptions in thermal and transport science. Submitted to the Journal of Engineering Education. (2008)

[8] Prince, M.J., Vigeant, M. A., and Nottis, K. E. K.: The use of inquiry-based activities to repair student misconceptions related to heat, energy, and temperature. American Socienty for Engineering Education (2012)

[9] Pathare, S.R. and Pradhan, H. C.: Students' misconceptions about heat transfer mechanisms and elementary kinetic theory. Vol. 45 (6), pp. 629-634. Physics Education (2010) 
[10] Miller, R. L., Streveler, R. A., Yang, D., Santiago-Roman, A. I. : Identifying and repairing student misconceptions in thermal and transport science: Concept inventories and schema training studies. Vol. 45 (3) pp. 203-210. Fundamental Research in Engineering Education (2011)

[11] Chiou, G.-L. and Anderson, O. R.: A study of undergraduate physics students' understanding of heat conduction based on mental model theory and an Ontology-Process Analysis. Vol. 94, pp. 825854. Science Education (2010)

[12] Clement, J.: Model based learning as a key research area for science education. Vol. 22 (9) pp. 1041-1053. International Journal of Science Education (2000)

[13] Louca, L. T. \& Zacharia, Z. C.: Modeling-based learning in science education: Cognitive, metacognitive, social, material and epistemological contributions. Vol. 64 (4), pp. 471-492. Educational Review (2012)

[14] Sari, I. M. and Saepuzaman, D.: Proc. Int. Conf. On Innovation in Engineering and Vocational Education (ICIEVE) Atlantis Press (2015)

[15] Sari, I.M.: What do they know about heat and heat conduction? A case study to excavate preservice physics teachers' mental model in heat and heat conduction . Journal of Physics: Conference Series 812012090 (2017)

[16] Sari, I.M. Malik, A., Saepuzaman, D., Rusdiana, D., and Ramalis, T. R.: Pre-service physics teachers' mental models of heat conduction: a case study of the process-analogy of heat conduction. Journal of Physics: Conference Series 1157032073 (2019)

[17] Sari, I.M., Fauzi, D., Malik, A., Saepuzaman, D., Ramalis, T. R., Rusdiana, D.: Excavating the quality of vocational students' mental models and prediction on heat conduction. Journal of Physics: Conference Series 1204012042 (2019)

[18] Tiberghien, A., Vince, J. and Gaidioz, P.: Design-based research: case of a teaching sequence on mechanics.Vol. 31 (17) pp. 2275-2314. International Journal of Science Education. (2014)

[19] Anderson, T. and Shattuck, J.: Design-Based Research: A decade of progress in education research. Vol. 41 No. 1, pp. 16-25. Educational Researcher (2012)

[20] Goff, W. M. and Getenet, S.: Design-based research in Doctoral studies: adding a new dimebnsion to doctoral research. Vol. 12, pp. 107-121. International Journal of Doctoral Studies (2017)

[21] Santoso, A.: Studi Deskriptif Effect Size penelitian-penelitian di Fakultas Psikologi Universitas Sanata Dharma. Vol. 14 (1). Jurnal Penelitian (2010)

[22] Metlzer, D.: The relationship between mathematics preparationm and conceptual learning gains in physics: A possible hidden variable“ in diagnostic pretest scores. Vol. 70 (12), pp. 1259-1268. American Journal of Physics (2002)

[23] Hake, R. R.: Analyzing change/gain scores. American Educational Research Association's Division D, Measurement and Research Methodology (1999)

[24] Greca, I. M. and Moreira, M. A.: Mental models, conceptual models, and modelling. Vol. 22 (1) pp. 1-11. International Journal of Science Education (2000) 